\title{
THRUST ENHANCEMENT OF THE GASDYNAMIC MIRROR (GDM) FUSION PROPULSION SYSTEM
}

\author{
Terry Kammash and Myoung-Jae Lee \\ Department of Nuclear Engineering and \\ Radiological Sciences \\ The University of Michigan \\ Ann Arbor, MI 48109 \\ (313) 764-0205
}

\author{
David I. Poston \\ Los Alamos National Laboratory \\ MS K-551 \\ Los Alamos, NM 87545 \\ (505) $667-4336$
}

\begin{abstract}
The gasdynamic mirror propulsion system is a device that utilizes a magnetic mirror configuration to confine a hot plasma to allow fusion reactions to take place while ejecting a fraction of the energetic charged particles through one end to generate thrust. Because the fusion fuel is generally an isotope of hydrogen, e.g., deuterium or tritium, this propulsion device is capable of producing very large specific impulses (e.g., 200,000 seconds) but at modest thrusts. Since large thrusts are desirable, not only for reducing travel time but also for lifting sizable payloads, we have examined methods by which GDM's thrust could be enhanced. The first consists of utilizing the radiation generated by the plasma, namely bremsstrahlung and synchrotron radiation, to heat a bydrogen propellant which upon exhausting through a nozzle produces the additional thrust. We asses the performance in this case by using an ideal model that ignores heat transfer considerations of the chamber wall, and one that takes into account heat flow and wall temperature limitations. We find in the case of a DT burning plasma that although thrust enhancement is significant, it was more than offset by the large drop in the specific impulse and a concomitant increase in travel time. The second method consisted of not altering the original GDM operation, but simply increasing the density of the injected plasma to achieve higher thrust. It is shown that the latter approach is more effective since it is compatible with improved performance in that it reduces trip time but at the expense of larger vehicle mass. For a $\mathrm{D}-\mathrm{He}^{3}$ burning device the use of hydrogen to enhance thrust appears to be more desirable since the radiated power that goes into heating the hydrogen propellant is quite large.
\end{abstract}

\section{INTRODUCTION}

One of the most promising propulsion systems that could be utilized in space exploration is the gasdynamic mirror (GDM) fusion device (Kammash and Lee 1995a). It utilizes a simple magnetic mirror geometry which provides adequate confinement for a hot plasma to undergo fusion reactions while allowing a fraction of its charged particle population to escape through the end to generate thrust. Unlike the device which was studied for decades a potential terrestrial power reactor whose the plasma was deemed to be collisionless, GDM will operate at a significantly high density to make the collision mean free path much larger than the machine length. Under these conditions the plasma behaves much like a fluid and the escape of the plasma from the system is analogous to the flow of a gas into a vacuum from a vessel with a hole. It can readily be shown (Kammash and Lee 1995a) that the particle lifetime in such a device is given by

$$
\tau=R L v_{t h}
$$

where $R$ is the mirror ratio seen by the plasma, $L$ the length and $\mathrm{v}_{t h}$ the particle's mean velocity. When an appropriate set of conservation equations are used to evaluate the performance of the system, it can be shown (Kammash and Lee 1995a) that the length of the device scales with plasma parameters in accordance with the relation

$$
L=\frac{E_{\text {in }}-2 T}{n R c_{0}\left[p_{0}+s_{0} T^{32}-\frac{1}{4}\{\sigma \gamma)\left(E_{\text {in }}+E_{0}\right) T^{-112}\right]}
$$

where $E_{\mathrm{m}}$ is the energy of the injected particles, $T$ the plasma temperature, $n$ the plasma density, $R$ the mirror ratio noted earlier, $E_{0}$ the fusion energy that remains in the plasma to heat it, and $c_{0}, p_{0}$ and $s_{0}$ are constants. The value of Ein can be established by first noting that the injected power $p_{i}$ can be expressed in terms of the fusion power $P_{f}$ through the $Q$-value of the reactor, namely, 


$$
\frac{P_{f}}{P_{l}}=\frac{n^{2}(\sigma) E_{f} / 4}{n E_{l n} / \tau}=Q
$$

where $\{0 v\rangle$ is the velocity-averaged fusion reaction cross section and $E_{f}$ the energy produced by the fusion reaction which in the case of deuterium-tritium (DT) is $17.6 \mathrm{MeV}$. The above analysis reveals that the length of the device decreases with increasing density and with increasing mirror ratio. It also sbows that the length decreased with decreasing $Q$ and appears to reach a minimum in all cases at a temperature of about $10 \mathrm{keV}$. These facts show dramatically why GDM is particularly suitable as a propulsion device because, unlike the terrestrial power reactor, it requires a relatively small energy magnification factor, $Q$, which is easier to obtain, and can have the size and symmetry that allows for ease of assembly on earth or in space. The parameters of a seemingly attractive propulsion GDM are shown in Table 1 for two fuel cycles. In obtaining the total mass of the vehicles shown in Table 1 we assumed that the magnets needed to confine the plasma are superconducting magnets, and as a measure of the effectiveness of the device as a propulsion system we used the parameters to compute a round trip journey to Mars. In calculating the travel time we employed a constant thrust, constant specific impulse continuous burn acceleration/deceleration type of trajectory (Kammash 1995b) when the distance from Earth to Mars is the linear distance measured when Mars lines itself between the earth and the sun (approximately every 26 months). The most glaring property of the GDM propulsion system is the very high specific impulse it generates while producing a modest thrust by comparison. Since large thrusts are desirable not only to reduce trip times, but also to lift sizable payloads we focus our attention in this paper on methods for potential thrust enhancement. Specifically, we examined the utilization of a portion of the fusion energy produced by the plasma in heating a hydrogen propellant that can be exhausted through a nozzle to generate the additional thrust. We also investigate thrust enhancement through modification of operating parameters without resort to supplementary propellant.

TABLE 1. GDM Propulsion Device Parameters.

\begin{tabular}{|l|r|r|}
\hline \multicolumn{1}{|c|}{ Parameter } & \multicolumn{1}{c|}{ D-T } & \multicolumn{1}{c|}{ D-He ${ }^{3}$} \\
\hline Plasma density, (m ${ }^{-3}$ ) & $1.0 \times 10^{2}$ & $1.0 \times 10^{22}$ \\
Plasma temperature, (keV) & 10 & 60 \\
Plasma radius, (m) & .05 & .05 \\
Plasma length, (m) & 44 & 1297 \\
Gain factor & 1.222 & 1.222 \\
Fusion power, (MW) & $2.730 \times 10^{3}$ & $5.675 \times 10^{4}$ \\
Bremsstrahlung power, (MW) & $5.817 \times 10^{1}$ & $1.703 \times 10^{4}$ \\
Synchrotron rad. power, (MW) & $1.894 \times 10^{1}$ & $4.205 \times 10^{4}$ \\
Neutron power, (MW) & $2.183 \times 10^{3}$ & $6.213 \times 10^{3}$ \\
Thrust, (N) & $2.512 \times 10^{3}$ & $1.437 \times 10^{4}$ \\
Thrust power, (MW) & $1.351 \times 10^{3}$ & $1.894 \times 10^{4}$ \\
Injection power, (MW) & $2.233 \times 10^{3}$ & $4.643 \times 10^{4}$ \\
Total vehicle mass, (mT) & 422 & 4434 \\
Specific power, (kW/kg) & 13.40 & 6.28 \\
Specific impulse, (s) & $1.268 \times 10^{5}$ & $3.106 \times 10^{5}$ \\
Round trip to Mars, (days) & 171 & 363 \\
\hline
\end{tabular}

\section{RADIATION HEATING OF HYDROGEN PROPELLANT}

We note from Table 1 that approximately $77 \mathrm{MW}$ of power are radiated by the plasma in the DT burning GDM, while about $59 \mathrm{GW}$ are generated in the $\mathrm{D}-\mathrm{He}^{3}$ case. It is interesting, therefore, to see if such power can be utilized in heating a hydrogen propellant to produce additional thrust. We address this question first by using a simple model which assumes that all the radiation is absorbed by a hydrogen stream that is injected into the 
reactor chamber at a certain inlet temperature and allowing no heat flow to the wall or mixing between plasma and hydrogen as illustrated in Fig ure. 1.

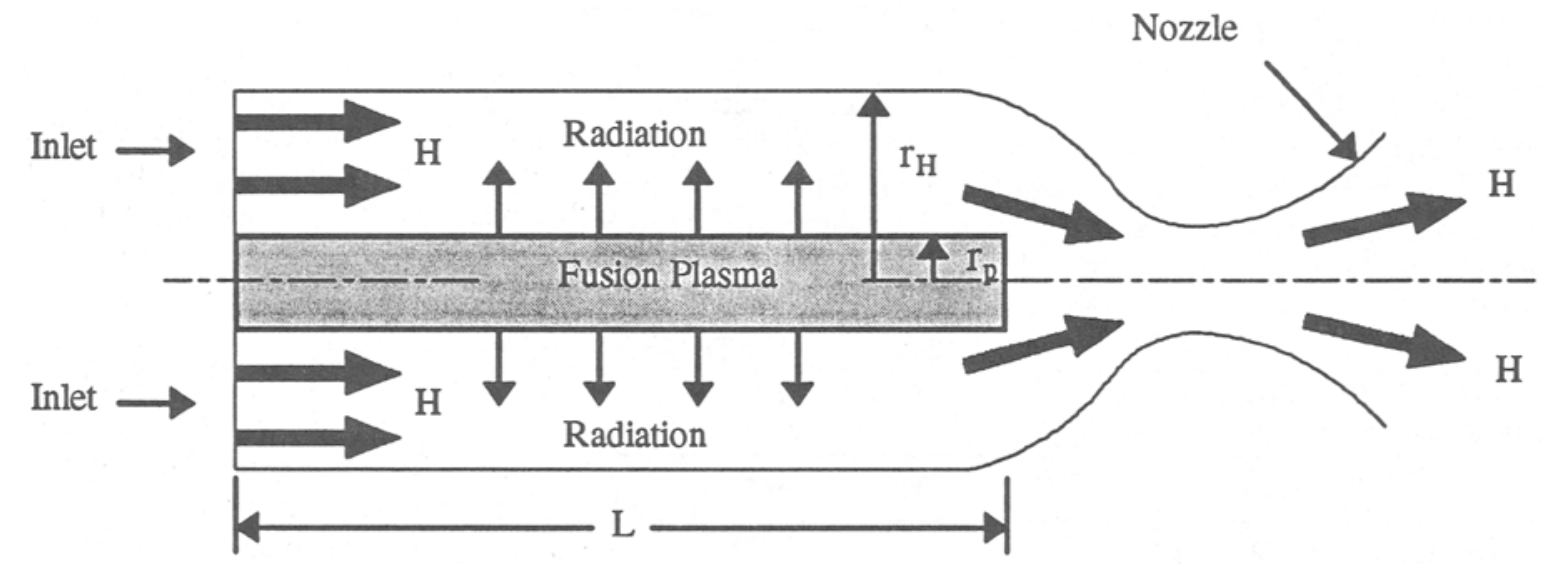

FIGURE 1. Thrust-Enhanced GDM Configuration.

When the gas emissivity is ignored the temperature change is given by (Deissler 1964)

$$
\rho C_{P} u \frac{d T_{H}}{d x}=P_{r}
$$

where $\rho$ is the gas mass density, $C_{P}$ the specific heat, $u$ the flow velocity, $P_{r}$ the radiated power per unit volume or the heat source and $d T / d x$ the temperature change in the direction of the flow. Noting that $\rho=n_{B} m_{H}$ where $n_{H}$ is the particle density, and $m_{H}$ the hydrogen molecular mass, Equation (4) can be rewritten as

$$
C_{P} \frac{d T_{H}}{d x}=\frac{P_{r}}{n_{H} m_{H}}
$$

which upon integration becomes

$$
T_{H}=T_{H 0}+\frac{\tau_{H} P_{r}}{C_{P} n_{H} m_{H}}
$$

where $T_{H o}$ is the inlet temperature and $\tau_{H}$ the propellant residence time in the chamber given by

$$
\tau_{H}=\frac{L}{u} \sim \frac{L}{\sqrt{T_{H}}}
$$

We apply the above analysis to the GDM case and obtain the results given in Table 2 for an inlet temperature of $3000 \mathrm{~K}$ and two fuel cycles, namely, DT and D-He ${ }^{3}$.

In both instances the effective thrust of the engine is increased significantly relative to the un-enhanced case but because of the much larger hydrogen mass flow rate ( 3 and $270 \mathrm{~kg} / \mathrm{s}$ ) compared to the plasma flow rate of about $0.002 \mathrm{~kg} / \mathrm{s}$, the effective specific impulse is decided almost totally by the hydrogen propellant and that represented an almost two orders of magnitude drop. When both of these factors are taken into account in calculating the round trip time to Mars we find an increase in travel time of about 325 days in the case of DT and 15 days in the D-He ${ }^{3}$ case. 
When a more realistic thermal hydraulic model (Poston and Kammash 1996) that takes into account heat flow to the wall where temperature is maintained at $3000 \mathrm{~K}$, the results shown in Table 3 are obtained. With this computational approach we have examined several cases where the hydrogen layer thickness was varied from 2 $\mathrm{cm}$ to $50 \mathrm{~cm}$ and calculated, among other things, the heat power to the wall. When compared to the results in Table 1 we see once again that the effective thrust is significantly increased but that it decreased with increasing layer thickness. While the power to the wall remained effectively the same, the effective specific impulse seemed to increase slightly with thickness up to a point $(25 \mathrm{~cm})$ then decline. In all cases, however, the effective specific impulse reflects a drop of two orders of magnitude relative to the un-enhanced case due primarily to the very small plasma mass flow rate.

TABLE 2. No Heat Transfer to Wall.

\begin{tabular}{|c|c|c|c|c|c|}
\hline $\begin{array}{c}\text { Fuel } \\
\text { Cycle }\end{array}$ & $\begin{array}{c}\text { Rad. Power } \\
(\mathrm{MW})\end{array}$ & $\begin{array}{c}\text { H-Flow Rate } \\
(\mathrm{kg} / \mathrm{s})\end{array}$ & Inlet Temp. (K) & H-Layer (m) & $\begin{array}{c}\text { Exit } \\
\text { Temp.(K) }\end{array}$ \\
\hline $\mathrm{D}-\mathrm{T}$ & 77 & 3.00 & 3000 & .05 & 4325 \\
\hline $\mathrm{D}^{-\mathrm{He}^{3}}$ & $59 \times 10^{3}$ & 270.00 & 3000 & .05 & 14218 \\
\hline
\end{tabular}

\begin{tabular}{|c|c|c|c|c|}
\hline $\begin{array}{c}\text { Fuel } \\
\text { Cycle }\end{array}$ & $\begin{array}{c}\text { Pre-Heat } \\
\text { Power }(\mathrm{MW})\end{array}$ & $\begin{array}{c}\text { Effective Thrust } \\
(\mathrm{kN})\end{array}$ & $\begin{array}{c}\text { Effective Specific Impulse } \\
(\mathrm{s})\end{array}$ & $\begin{array}{c}\text { Trip Time to } \\
\text { Mars (days) }\end{array}$ \\
\hline D-T & $1.746 \times 10^{2}$ & $24.48(2.512)^{*}$ & $8.32 \times 10^{2}\left(1.268 \times 10^{5}\right)$ & $496(171)$ \\
\hline D- $\mathrm{He}^{3}$ & $1.571 \times 10^{4}$ & $3.607 \times 10^{3}(14.37)$ & $1.36 \times 10^{3}\left(3.106 \times 10^{5}\right)$ & $284(269)$ \\
\hline
\end{tabular}

* quantities in parenthesis are for un-enhanced system.

TABLE 3. With Heat Transfer Considerations, DT Fuel Cycle.

\begin{tabular}{|c|c|c|c|c|}
\hline H-Layer (m) & $\begin{array}{c}\text { H-Mass Flow } \\
\text { Rate }(\mathrm{kg} / \mathrm{s})\end{array}$ & $\begin{array}{c}\text { H-Specific } \\
\text { Impulse (s) }\end{array}$ & Thrust (kN) & $\begin{array}{c}\text { Pre-Heat Power } \\
(\mathrm{MW})\end{array}$ \\
\hline .02 & 4.80 & 1052 & 49.5 & 237 \\
\hline .05 & 3.00 & 1117 & 32.8 & 148 \\
\hline .10 & 2.35 & 1164 & 26.8 & 116 \\
\hline .25 & 1.95 & 1200 & 22.9 & 96 \\
\hline .50 & 2.00 & 1195 & 23.4 & 99 \\
\hline
\end{tabular}

\begin{tabular}{|c|c|c|c|}
\hline H-Layer (m) & $\begin{array}{c}\text { Power to Wall } \\
(\mathrm{MW})\end{array}$ & $\begin{array}{c}\text { Maximum } \\
\text { Temperature (K) }\end{array}$ & $\begin{array}{c}\text { Average } \\
\text { Temperature (K) }\end{array}$ \\
\hline .02 & 7.5 & 3867 & 3454 \\
\hline .05 & 7.4 & 4364 & 3729 \\
\hline .10 & 7.4 & 4775 & 3930 \\
\hline .25 & 7.6 & 5259 & 4077 \\
\hline .50 & 7.7 & 5510 & 4058 \\
\hline
\end{tabular}

The pre-heat power is the power required to heat the hydrogen up to its inlet temperature of $3000 \mathrm{~K}$. This can be achieved by regeneratively cooling the nozzle or other system components - provided there is enough power. In the absence of such power the fusion $Q$-value should be increased to accommodate this need. As both Tables 2 and 3 reveal, the majority of the additional thrust is due to the pre-heating of the hydrogen in the DT case and to a much lesser extent in the $\mathrm{D}-\mathrm{He}^{3}$ case. In all instances additional thrust is gained due to flow in the chamber where heating by the radiated power takes place. The maximum attainable temperature of the hydrogen is set by the 
heat flux, wall temperature, and hydrogen layer thickness. It is independent of flow rate because as soon as the hydrogen reaches its maximum temperature it is "saturated" with heat such that additional energy input flows through the hydrogen to the wall. The hydrogen temperature at the wall is fixed by the wall temperature, the temperature gradient is fixed by the heat flux, and the maximum temperature is determined by the gradient and the layer thickness.

The results given in Table 3 were calculated by setting the limit on power conducted to the wall at $10 \%$ of the total power input to the hydrogen. Lower flow rates will result in only slightly higher hydrogen specific impulse but lower thrust in proportion to the flow rate. It appears on the basis of this thermal hydraulic model that the only way to get significantly higher specific impulse out of the hydrogen is to make the radial heat flux higher same power over a shorter core length. Increasing the wall temperature is not really an option, and increasing the hydrogen layer thickness helps only marginally. In fact if it is made tow large it will have a negative effect as can be seen from the $50 \mathrm{~cm}$ case shown in the Table 3 .

\section{THRUST ENHANCEMENT BY PLASMA DENSITY INCREASE}

A more direct approach to increasing thrust in GDM is to inject the hot plasma at higher densities. One order of magnitude increase in the density results in a similar increase in thrust and for the DT case given in Table 2 the result is almost identical to that of using a hydrogen propellant, but with the distinct advantage of not degrading the specific impulse. This salutary effect is not, however, without penalty since increasing the density from $10^{16}$ $\mathrm{cm}^{-3}$ to $10^{17} \mathrm{~cm}^{-3}$ results in about six fold increase in the total mass of the vehicle and an order of magnitude increase in injected power. The redeeming factor in the higher density case is nevertheless an order of magnitude shorter vehicle and a decrease in a Mars trip time of about 40 days, i.e., from 171 to 131.

\section{CONCLUSION}

We have examined in this paper means by which the thrust in the gasdynamic mirror fusion propulsion system can be enhanced. The first approach consisted of utilizing a hydrogen propellant that is introduced into the reactor chamber at very high pressure and allowed to be heated by the radiation emitted by the fusion plasma then exhausted through a nozzle to provide the additional thrust. Using a simple model which ignores heat flow to the wall, and a comprehensive thermal hydraulic model that accounts for heat transfer and wall temperature limitations we calculated the additional thrust generated by the hydrogen propellant and its specific impulse. We find that the improvement in the thrust was more than offset by the sharp decline of the specific impulse of the engine and the corresponding travel time for a DT burning device. In the case of $\mathrm{D}-\mathrm{He}^{3}$ system where the radiated power was significantly larger, the change in the propulsive capability of GDM was quite minor although the total mass of the vehicle was much larger than its DT counterpart. Although incapable of predicting the heat transfer characteristics the simple model was sufficiently reliable in predicting the propulsion performance of a GDM that utilizes a hydrogen propellant for thrust enhancement.

The other approach consisted of simply increasing the injected plasma density into GDM where equally as effective results can be achieved. Although this approach resulted also in larger vehicle mass, it also resulted in a much shorter engine with a shorter trip time. Based purely on performance one would tend to conclude that the "increasing plasma density" approach is more desirable since it leads to no degradation of propulsion capability of GDM. On the other hand, engineering considerations and wall design may necessitate the use of a buffer zone to moderate the heat flow and that may make the use of hydrogen propellant inevitable.

\section{Acknowledgments}

This work was supported in part by NASA under contract NAG8-1293 with the University of Michigan. 


\section{References}

Deissler, R. G. (1964), "Diffusion Approximation for thermal Radiation in Gases with Jump Boundary Conditions," Journal of Heat Transfer, May 1964, 240-246.

Kammash, T. and M. J. Lee (1995a) "Gasdynamic Fusion Propulsion System for Space Exploration," J. Propulsion and Power 11: 544-553.

Kammash (1995b) Fusion Energy in Space Propulsion, AIAA Progress in Aeronautics and Astronautics 167(1): 146.

Poston, D. I. and T. Kammash (1996), "A Computational Model for an Open-Cycle Gas Core Nuclear Rocket," Nuclear Sciences and Engineering, 122, 32.

\begin{tabular}{|c|c|c|c|}
\hline$c_{0}:$ & constant, Equation 2 & $P_{r}:$ & radiated power, $(\mathrm{MW})$ \\
\hline$C_{p}:$ & specific heat at constant pressure, $(\mathrm{J} / \mathrm{kg} * \mathrm{~K})$ & $Q:$ & fusion energy multiplication \\
\hline$E_{0}:$ & fusion energy deposited in plasma, $(\mathrm{keV})$ & $R:$ & plasma mirror ratio \\
\hline$E_{f}$ & energy produced by fusion reaction, $(\mathrm{keV})$ & $s_{0}:$ & constant, Equation 2 \\
\hline$E_{m}:$ & energy of injected plasma, $(\mathrm{keV})$ & $T:$ & plasma temperature, $(\mathrm{keV})$ \\
\hline$L:$ & length of engine, (m) & $T_{0}:$ & hydrogen inlet temperature, $(\mathrm{K})$ \\
\hline$m_{H}:$ & mass of hydrogen molecule, $(\mathrm{kg})$ & $T_{H}:$ & hydrogen temperature, $(\mathrm{K})$ \\
\hline$n:$ & plasma particle density, $\left(\mathrm{m}^{-3}\right)$ & $u:$ & hydrogen flow velocity, $(\mathrm{m} / \mathrm{s})$ \\
\hline$n_{H}:$ & hydrogen molecular density, $\left(\mathrm{m}^{-3}\right)$ & $v_{t h}$ & plasma particle thermal velocity, $(\mathrm{m} / \mathrm{s})$ \\
\hline$p_{0}:$ & constant, Equation 2 & $\rho:$ & hydrogen gas mass density, $\left(\mathrm{kg} / \mathrm{m}^{3}\right)$ \\
\hline$P_{\dot{r}}$ & fusion power, (MW) & $\tau:$ & plasma confinement time, $(s)$ \\
\hline$P_{i}$ & injected power, (MW) & $\tau_{\mu}:$ & hydrogen residence time, (s) \\
\hline
\end{tabular}

